\title{
Estudio Monte Carlo de un Ferrimagneto de Ising Mixto con Diferentes Anisotropías
}

\author{
Nicolás A. De La Espriella, Gladys R. Casiano, César Ortega \\ Universidad de Córdoba. Departamento de Física y Electrónica. Grupo Gamasco. Facultad de \\ Ciencias Básicas. Carrera 6 No. 76-103. Córdoba, Colombia. e-mail: ndelae52@gmail.com
}

Recibido Oct. 06, 2011; Aceptado Nov. 28, 2011; Versión Final recibida Dic. 30, 2011

\begin{abstract}
Resumen
Mediante simulaciones de Monte Carlo, se analizan las propiedades magnéticas de un modelo ferrimagnético de Ising mixto, con espines $S= \pm 3 / 2, \pm 1 / 2$ y $\sigma= \pm 5 / 2, \pm 3 / 2, \pm 1 / 2$ distribuidos sobre una red cuadrada, con diferentes anisotropías. Se supuso que la interacción de intercambio a primeros vecinos, $J_{1}$, entre espines $S$ y $\sigma$ es antiferromagnética $\left(J_{1}<0\right)$. También, se consideró el efecto de las intensidades de las anisotropías de ión simple, debidas a los campos cristalinos de las subredes $S$ y $\sigma, D_{S}$ y $D_{\sigma}$, respectivamente. Se investigó la existencia y dependencia de las temperaturas de compensación del modelo con respecto a las anisotropías de ión simple. Fijando el parámetro $D_{s}$ y variando la intensidad de $D_{\sigma}$, aparecen posibles transiciones de fase de primer orden. El análisis de las temperaturas críticas se obtiene a través de los máximos del calor específico del sistema. Los diagramas de fase a temperaturas finitas se obtienen en el plano temperatura-anisotropía.
\end{abstract}

Palabras clave: modelo de Ising, Monte Carlo, temperaturas de compensación, temperaturas críticas, anisotropías de ión simple

\section{Monte Carlo Study of a Mixed Ising Ferrimagnet with Different Anisotropies}

\begin{abstract}
Using Monte Carlo simulations, the magnetic properties of a mixed Ising ferrimagnetic model with spins $S= \pm 3 / 2, \pm 1 / 2$ y $\sigma= \pm 5 / 2, \pm 3 / 2, \pm 1 / 2$ distributed on a square lattice with different anisotropies was analyzed. It was assumed that the exchange interaction to nearest neighbors, $J_{1}$, between spins $S$ and $\sigma$, is antiferromagnetic $\left(J_{1}<0\right)$. Also, it was considered that the effect of the intensities of the single-ion anisotropies, due to the crystalline fields of the sublattices $S$ and $\sigma, D_{S}$ and $D_{\sigma}$ respectively. The existence and dependence of the compensation temperature in the model with respect to the single-ion anisotropies was also studied. By fixing the parameter $D_{s}$ and varying the intensity of $D_{\sigma}$ it probable phase transitions of first order appear. The analysis of the critical temperatures is obtained through the maximum of the specific heat of the system. Phase diagrams at finite temperatures are obtained in the temperature-anisotropy plane.
\end{abstract}

Keywords: Ising Model, Monte Carlo, compensation temperatures, critical temperatures, single-ion anisotropies. 


\section{INTRODUCCIÓN}

En la actualidad se ha incrementado el interés por el estudio de los materiales ferrimagnéticos, debido a su importancia y utilidad en muchas aplicaciones tecnológicas, dentro las que se destacan las grabaciones termomagnéticas. Se ha dedicado gran empeño al estudio de los fenómenos críticos de estos materiales, ya que hacen parte de algunos compuestos magnéticos moleculares, para cuyo diseño y síntesis se emplean técnicas de la Química Orgánica (Blundell y Pratt, 2004; Gatteschi y Sessoli, 2004).

Los ferrimagnetos son materiales compuestos por varias subredes con momentos magnéticos distintos, que interactúan antiferromagnéticamente. El estudio teórico de estos materiales requiere un gran esfuerzo, para clarificar su interesante, y muchas veces, inusual comportamiento magnético (Keskin y Ertas, 2009; Keskin y Kantar, 2010). Dentro de las características más relevantes de los ferrimagnetos, está la exhibición de temperaturas de compensación, $T_{\text {Comp. }}$. Temperaturas menores que la temperatura crítica $\left(T_{C}\right)$, que surgen bajo ciertas condiciones, cuando las magnetizaciones de las distintas subredes se cancelan (se compensan), de modo que la magnetización total del sistema es cero. La aparición de puntos de compensación es de gran importancia tecnológica, puesto que a $T=T_{\text {Comp }}$ sólo se requiere un pequeño campo conductor para invertir la magnetización resultante (Ekiz, 2005). Además, en este punto la coercitividad del material se incrementa dramáticamente permitiendo la formación de pequeños dominios magnéticos estables (Buendía y Machado, 2000), lo cual facilita los procesos de escritura y borrado en memorias magneto-ópticas de alta densidad (Deviren et al., 2009a; Hurtado y Buendía, 2003), donde los cambios de temperatura se hacen calentando localmente la película magnética, mediante un rayo láser (Kiwi, 2001). A pesar del creciente trabajo experimental sobre los ferrimagnetos, y en general sobre los materiales magnéticos moleculares, su comprensión aún sigue siendo un tema abierto que requiere elementos de diversas áreas de las ciencias básicas, por ello se necesita una contraparte teórica para el estudio y análisis magnético de esta nueva clase de materiales.

Los modelos de Ising mixtos se emplean en el magnetismo molecular, como una herramienta para la comprensión teórica de las propiedades magnéticas de muchos compuestos moleculares, debido a que a través de ellos se pueden calcular exactamente cantidades físicas observables. Muchos trabajos teóricos relacionados con el estudio de sistemas de multicapas magnéticas, se desarrollan con modelos de Ising mixtos (Ekiz, 2008).

Con el propósito de optimizar el diseño de los procesos, en el campo de los materiales moleculares ferrimagnéticos, se hace imprescindible entender el mecanismo que fundamenta las inusuales propiedades magnéticas de este tipo de sistemas. Particularmente, comprender el efecto de las interacciones de intercambio entre las diferentes moléculas que forman el compuesto, y de qué manera estas interacciones afectan $T_{c}$ y $T_{\text {comp. }}$. Por ejemplo, observamos como el solo hecho de sustituir un metal por otro, en un compuesto, hace que surjan nuevas propiedades magnéticas (Coronado y Gatteshi, 2006). Los modelos de Ising mixtos juegan un papel importante en la simulación de dichas propiedades (Keskin y Polat, 2009; Behera et al., 2009). Se han realizado, por diversos métodos, diferentes estudios sobre estos modelos compuestos por dos o más subredes de espines mixtos (De La Espriella y Buendía, 2011; De La Espriella y Buendía, 2010; Deviren y Keskin, 2010; Yessoufou et al., 2009; Albayrak e Yigit, 2007; Buendía y Villarroel, 2007; Zhang et al., 2005; Htoutou et al., 2004; Zaim et al., 2009; Ekiz, 2008; Ekiz, 2005; Albayrak e Yigit, 2006).

El estudio de sistemas de Ising mixtos, con espines de valores altos, sigue siendo un tema relevante debido a que son atractivos por su rica variedad de fenómenos multicríticos (Albayrak e Yigit, 2007; Deviren et al., 2009b). Dentro de dichos sistemas, encontramos el modelo de espines mixtos ( $S=3 / 2, \sigma=5 / 2$ ), que es uno de los menos estudiados ( $\mathrm{Li}$ et al., 2006; Yessoufou et al., 2009), por ello resulta interesante analizarlo. Además de la importancia del modelo, con respecto al estudio y caracterización de magnetos moleculares (Albayrak e Yigit, 2007; Zhang et al., 2005), también parece que es responsable de las propiedades magnéticas de ciertas proteínas hemo- 
férricas conocidas como ferricitocromos c', cuyo rol fundamental es coadyuvar al transporte de oxígeno en la sangre (Weiss et al., 2006).

Hasta donde conocemos, el modelo $(S=3 / 2, \sigma=5 / 2$ ), en la mayoría de los casos, se ha estudiado con aproximaciones tipo campo medio y métodos perturbativos, sugiriéndose que el sistema posee un interesante comportamiento magnético que incluye transiciones de primer y segundo orden, $T_{\text {Comp }}$, y comportamientos reentrantes (Albayrak e Yigit, 2006; Yessoufou et al., 2009; Albayrak e Yigit, 2007). Es importante establecer que estas propiedades son características del modelo, y no un resultado de la aproximación que se usa. Existen casos de algunos modelos, donde las aproximaciones de campo medio predicen la existencia de puntos de compensación, en los que simulaciones posteriores con métodos no perturbativos (Monte Carlo) han demostrado que no los poseen (Figueiredo et al., 2004; De La Espriella y Buendía, 2011).

En este trabajo desarrollamos simulaciones numéricas, mediante el método de Monte Carlo, para investigar las propiedades magnéticas de un modelo de Ising mixto de espines $(S=3 / 2, \sigma=5 / 2$ ), con distintas anisotropías.

\section{FORMULACIÓN DEL MODELO Y LA SIMULACIÓN NUMÉRICA}

Consideramos un modelo de Ising formado por dos subredes cuadradas intercaladas de espines $S$ $= \pm 3 / 2, \pm 1 / 2$ y $\sigma= \pm 5 / 2, \pm 3 / 2, \pm 1 / 2$, con diferentes anisotropías de ión simple, que actúan sobre los espines $S$ y $\sigma$, respectivamente. El sistema está caracterizado por el Hamiltoniano de la forma:

$$
H=-J_{1} \sum_{<n n>} S_{i} \sigma_{j}-D_{S} \sum_{i} S_{i}^{2}-D_{\sigma} \sum_{j} \sigma_{j}^{2}
$$

$J_{1}$ representa la interacción de intercambio entre primeros vecinos $(<n n>), D_{S}$ y $D_{\sigma}$ son los campos cristalinos de cada subred, responsables de las anisotropías del sistema. La primera suma se efectúa sobre todos los pares de espines con interacción a primeros vecinos, es decir, entre los sitios con espines $S=3 / 2$ y $\sigma=5 / 2$, y las sumas $\Sigma_{i}$ y $\Sigma_{j}$ se realizan sobre todos los sitios de espines de las subredes $S$ y $\sigma$, respectivamente. Para realizar los cálculos tomamos una red de tamaño $L=60$, con condiciones de borde periódicas. Los parámetros están en unidades de energía. Seleccionamos el parámetro $J_{1}<0$, puesto que el acoplamiento entre primeros vecinos es antiferromagnético. El modelo se simula mediante algoritmos de Monte Carlo, que son métodos confiables para el estudio de este tipo de sistemas de Ising (De La Espriella y Buendía, 2011; Zukovic y Bobák, 2010; Selke y Oitmaa, 2010). Los datos se generan con 50000 pasos de Monte Carlo por sitio, después de descartar los primeros 5000 pasos. Las barras de error se calculan agrupando todas las medidas en 10 bloques y tomando su desviación estándar. Definimos $\beta=1 / k_{B} T$.

En la simulación se calcula el calor específico por sitio, $C$,

$$
C=\frac{\beta^{2}}{L^{2}}\left(<H^{2}>-<H>^{2}\right)
$$

las magnetizaciones de las subredes por sitio $\left(M_{S}, M_{\sigma}\right)$, y la magnetización total por espín, $M_{T}$ :

$$
M_{S}=\frac{2}{L^{2}}\left(<\sum_{i} S_{i}>\right) \quad M_{\sigma}=\frac{2}{L^{2}}\left(<\sum_{j} \sigma_{j}>\right) \quad M_{T}=\frac{M_{S}+M_{\sigma}}{2}
$$

Una forma eficiente de ubicar la temperatura de compensación ( $T_{\text {Comp }}$ ), usando los datos Monte Carlo, es hallando el punto de corte de los módulos $\left|M_{S}\right|$ y $\left|M_{\sigma}\right|$, es decir,

$\left|\mathrm{M}_{\mathrm{S}}\left(T_{\text {Comp }}\right)\right|=\left|\mathrm{M}_{\sigma}\left(T_{\text {Comp }}\right)\right|$ 
con las condiciones

$\operatorname{sign}\left(\mathrm{M}_{\mathrm{S}}\left(T_{\text {Comp }}\right)\right)=-\operatorname{sign}\left(\mathrm{M}_{\sigma}\left(T_{\text {Comp }}\right)\right) \quad$ y $\quad T_{\text {Comp }}<T_{C}$

\section{RESULTADOS Y DISCUSIÓN}

En la figura 1 se exhibe el diagrama de fase a $T=O K$, calculado en forma exacta, relacionado con los campos cristalinos $D_{S}$ y $D_{\sigma}$, que interaccionan con las subredes $S$ y $\sigma$, que conforman el sistema. El estado base del Hamiltoniano que describe el sistema es traslacionalmente invariante, por ello, de acuerdo a la conjetura de Luttinger y Tisza, una celda $2 \times 2$ es suficiente para incluir todos los posibles estados base del modelo (Karl, 1973). Se aprecian 6 regiones con 9 rectas multifases, y 4 puntos donde coexisten tres fases. El diagrama es muy parecido al reportado por Yessoufou (Yessoufou et al., 2009), y su topología es independiente del signo de $J_{1}$.

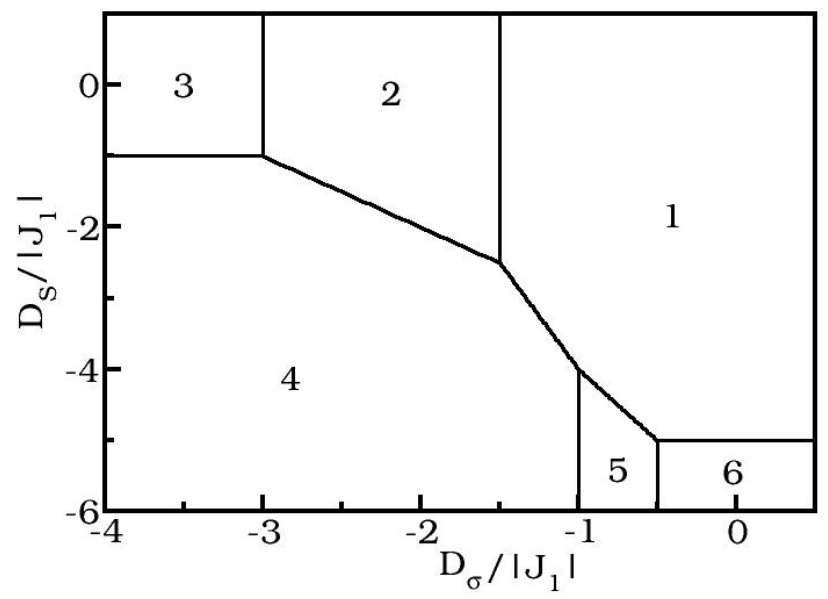

Fig. 1: Diagrama de fase a $T=O K$ para el modelo $J_{1}-D_{S}-D_{\sigma \cdot}\left|J_{1}\right|=1$.

La celda $2 \times 2$, que forma las configuraciones de estados base, tiene dos espines tipo $S$ y dos tipo $\sigma$, y sus energías, calculadas numéricamente, se muestran en la tabla 1.

Tabla 1: Energías de estados base del modelo $J_{1}-D_{S}-D_{\sigma} \cdot\left|J_{1}\right|=1$.

\begin{tabular}{|c|c|c|c|}
\hline \multicolumn{2}{|c|}{ Estado base: $\left|\begin{array}{ll}S & \sigma \\
\sigma & S\end{array}\right|$} & Energía & \multirow{2}{*}{$\begin{array}{c}\text { Región } \\
1\end{array}$} \\
\hline $\begin{array}{l}\mp 3 / 2 \\
\pm 5 / 2\end{array}$ & $\begin{array}{l} \pm 5 / 2 \\
\mp 3 / 2\end{array}$ & $-30\left|J_{1}\right|-4.5 D_{S}-12.5 D_{\sigma}$ & \\
\hline $\begin{array}{l}\mp 3 / 2 \\
\pm 3 / 2\end{array}$ & $\begin{array}{l} \pm 3 / 2 \\
\mp 3 / 2\end{array}$ & $-18\left|J_{1}\right|-4.5 D_{S}-4.5 D_{\sigma}$ & 2 \\
\hline $\begin{array}{l}\mp 3 / 2 \\
\pm 1 / 2\end{array}$ & $\begin{array}{l} \pm 1 / 2 \\
\mp 3 / 2\end{array}$ & $-6\left|J_{1}\right|-4.5 D_{S}-0.5 D_{\sigma}$ & 3 \\
\hline $\begin{array}{l}\mp 1 / 2 \\
\pm 1 / 2\end{array}$ & $\begin{array}{l} \pm 1 / 2 \\
\mp 1 / 2\end{array}$ & $-2\left|J_{1}\right|-0.5 D_{S}-0.5 D_{\sigma}$ & 4 \\
\hline $\begin{array}{l}\mp 1 / 2 \\
\pm 3 / 2\end{array}$ & $\begin{array}{l} \pm 3 / 2 \\
\mp 1 / 2\end{array}$ & $-6\left|J_{1}\right|-0.5 D_{S}-4.5 D_{\sigma}$ & 5 \\
\hline $\begin{array}{l}\mp 1 / 2 \\
\pm 5 / 2\end{array}$ & $\begin{array}{l} \pm 5 / 2 \\
\mp 1 / 2\end{array}$ & $-10\left|J_{1}\right|-0.5 D_{S}-12.5 D_{\sigma}$ & 6 \\
\hline
\end{tabular}


Se analiza el efecto de las anisotropías de ión simple, generadas por $D_{S}^{\prime}=D_{S} /\left|J_{1}\right|$ y $D^{\prime}{ }_{\sigma}=D_{\sigma} /\left|J_{1}\right|$ sobre las magnetizaciones, el calor específico, $T_{C}$ y $T_{\text {Comp. }}$.

Efectos de $D^{\prime} s$ y $D^{\prime} \sigma$

Las figuras 2 y 3 , muestran el comportamiento de $\left|\mathrm{M}_{\mathrm{T}}\right|$ para $D_{\sigma}^{\prime}=-1.95$ y $D_{S}^{\prime}$ variable $\left(D^{\prime}{ }_{S} \geq 0\right)$, en un rango de valores donde existe $T_{\text {comp. }}$. En todos los casos se observa que, cuando la temperatura se incrementa por encima de $T_{\text {comp }}$, la $\left|\mathrm{M}_{\mathrm{T}}\right|$ inicialmente crece desde cero hasta alcanzar un máximo local a una temperatura determinada, y luego decrece hasta hacerse cero en $T=T_{C}$. También, se observa que la $T_{\text {comp }}$ decrece con el incremento del campo $D_{\text {s }}$, hasta alcanzar un valor constante. El incremento del campo hace que el sistema adquiera un ordenamiento antiferromagnético a una menor temperatura. Aquí se forman pequeños dominios magnéticos estables, y la coercitividad del sistema se incrementa dramáticamente. La figura 2(a) representa el modelo particular $J_{1}-D_{\sigma}\left(D_{s}^{\prime}=0\right)$. Esto implica que el campo $D_{\sigma}^{\prime}$ contribuye radicalmente en la formación de la $T_{\text {Comp }}$.

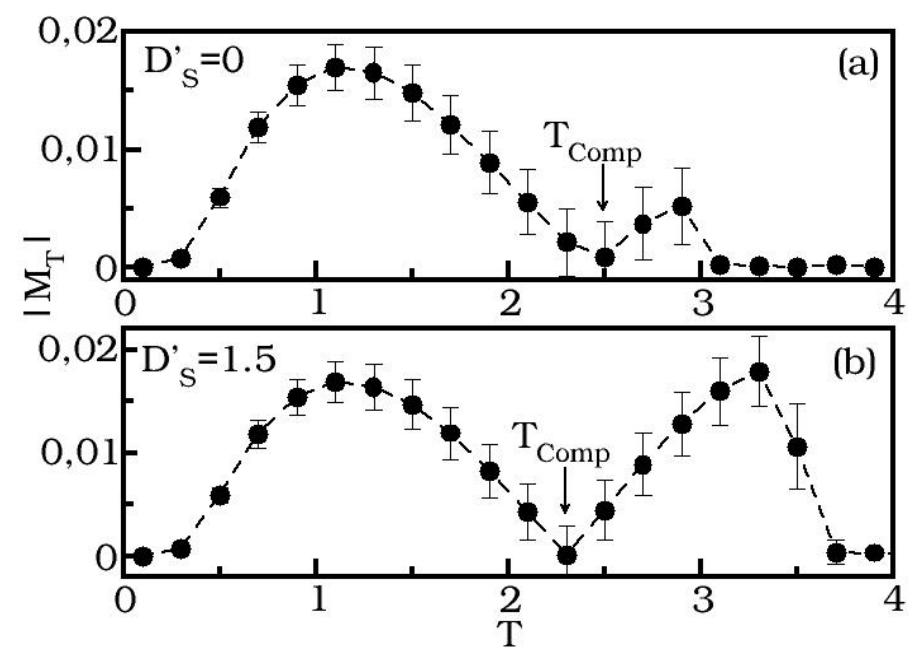

Fig. 2: Comportamiento de la magnetización total del sistema, $\left|\mathrm{M}_{\mathrm{T}}(\mathrm{T})\right|$, como una función de la temperatura, en una región con $T_{\text {comp. }} D_{\sigma}^{\prime}=-1.95$. $D_{s}^{\prime} \geq 0 . J_{1}<0$.

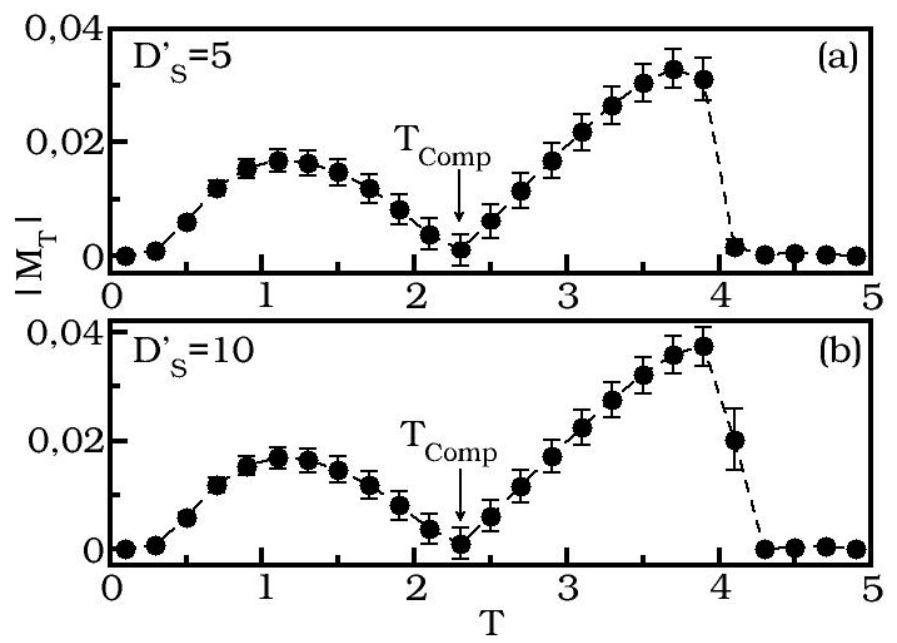

Fig. 3: Comportamiento de la magnetización total del sistema, $\left|\mathrm{M}_{\mathrm{T}}(\mathrm{T})\right|$, como una función de la temperatura, en una región con $T_{\text {Comp. }} D_{\sigma}^{\prime}=-1.95 . D_{S}^{\prime}>0 . J_{1}<0$.

Adicionalmente, se observa que al incrementar el campo $D^{\prime}$ s, la temperatura crítica se incrementa. El incremento del campo favorece que el sistema se mantenga en su fase ferrimagnética a temperaturas cada vez más altas. 
El fenómeno de compensación se puede entender mediante el análisis de la figura 4, que muestra el comportamiento de $\left|\mathrm{M}_{S}\right|$ y $\left|\mathrm{M}_{\sigma}\right|$, para el caso particular $D_{\sigma}^{\prime}=-1.95$ y $D_{S}^{\prime}=5$. Para $T<T_{\text {Comp }}$, la subred $S$ está más ordenada que la subred $\sigma$, y las magnetizaciones de las subredes tienen signos opuestos, pero su cancelación es aún incompleta, debido a que existe una magnetización residual en el sistema producida por $J_{1}$, la cual tiende a alinear los espines cercanos en direcciones opuestas. Cuando se incrementa la temperatura del sistema, la dirección de la magnetización residual puede cambiar, es decir, prevalece en ese instante la energía térmica, y muchos espines tienden a cambiar de dirección, hasta que en $T=T_{\text {comp }}$, las subredes se compensan, es decir, $\left|\mathrm{M}_{\mathrm{T}}\right|=0$. Para $T>T_{\text {Comp }}$, ambas subredes tienden a desordenarse, y en el instante $T=T_{C}$ el sistema estará completamente desordenado, y entra en la fase paramagnética. El efecto de compensación ocurre debido a las diferentes ratas a las que se desordena cada una de las subredes, y a la interacción antiferromagnética entre ellas.

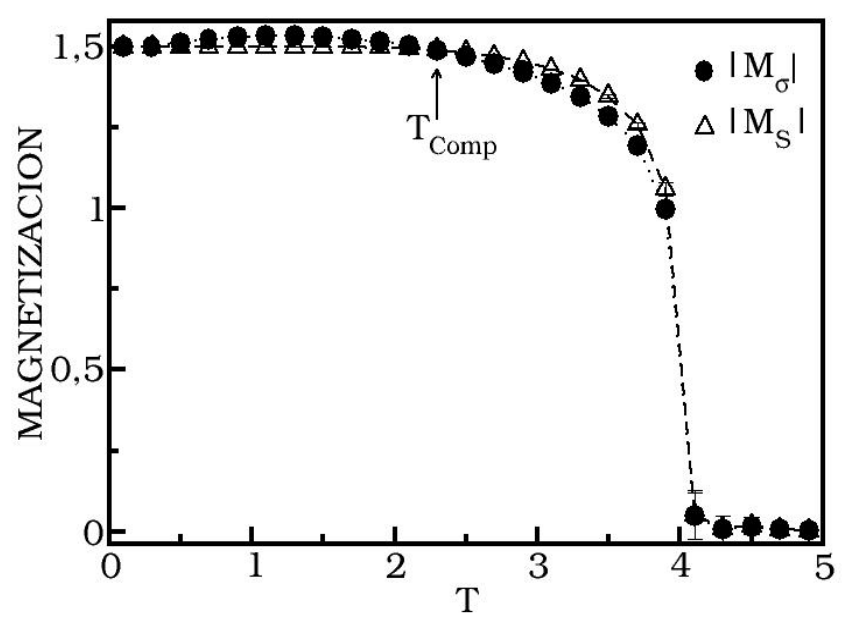

Fig. 4: Comportamiento de las magnetizaciones de las subredes, $\left|M_{\sigma}(T)\right|$ y $\left|M_{S}(T)\right|$, como una función de la temperatura, para el caso particular: $D_{\sigma}^{\prime}=-1.95 . D_{S}^{\prime}=5 . J_{1}<0$.

En la figura 5 , se muestra el comportamiento de $\left|\mathrm{M}_{S}\right|$ y $\left|\mathrm{M}_{\sigma}\right|$ para $D^{\prime}{ }_{S}=-1.5$ y $D^{\prime}{ }_{\sigma}=-2.5$. Estos valores de los campos, se encuentran sobre la línea de coexistencia de las fases 2 y 4 en el diagrama de fase a $T=O K$ (ver figura 1). Para este caso, se observa una posible transición de fase de primer orden en $T=T_{K}$, reflejada en el comportamiento de las magnetizaciones $\left|M_{S}\right|$ y $\left|\mathrm{M}_{\sigma}\right|$.

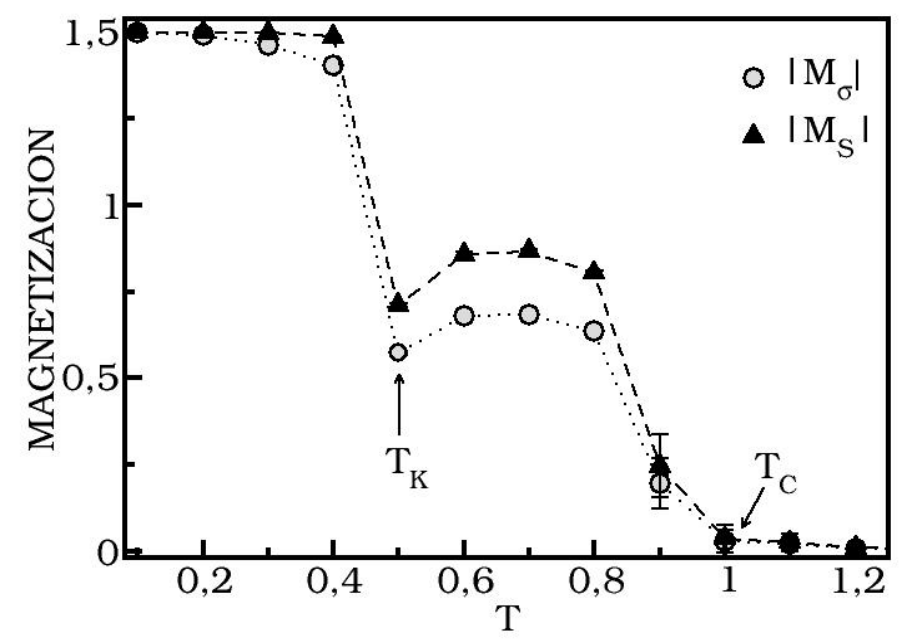

Fig. 5: Comportamiento de las magnetizaciones de las subredes, $\left|M_{\sigma}(T)\right|$ y $\left|M_{S}(T)\right|$. $T_{k}$ es la temperatura de la posible transición de primer orden. $D_{S}^{\prime}=-1.5 . D_{\sigma}^{\prime}=-2.5 . J_{1}<0$. 
También existe la posibilidad de que el sistema se halle en un estado metaestable, a estas temperaturas. Consideramos que estos cambios abruptos, pueden ocurrir para combinaciones de valores de los campos de anisotropías, cercanos a zonas de coexistencia de distintos estados base del sistema. En este caso, coinciden las energías de estado base de las regiones 2 y 4 , y las magnetizaciones total y de las subredes obtenidas a temperaturas bajas en la región 2 (ver tabla 1).

En la figura 6, se muestra el comportamiento de las magnetizaciones de las subredes, y de la magnetización total, para $D_{S}^{\prime}=-100$ y $D_{\sigma}^{\prime}=-1.95$. En este caso, donde el campo de la subred $S$ es grande comparado con $D_{\sigma}^{\prime}$, las curvas de $\left|M_{\sigma}(T)\right|$ y $\left|M_{S}(T)\right|$ exhiben un comportamiento interesante. Se aprecia que cuando se incrementa la temperatura a partir de cero, la magnetización de la subred $S$ decae desde su valor de saturación $\left|\mathrm{M}_{S}(T)\right|=1$, mientras que la magnetización de la subred $\sigma$ decrece desde $\left|\mathrm{M}_{\sigma}(T)\right|=1.5$. Cuando $T=T_{K}$, las magnetizaciones decrecen a 0.5 discontinuamente, experimentando una posible transición de fase de primer orden. Se nota también, que la temperatura crítica, $T_{C}$, disminuye ostensiblemente con respecto al caso para $D_{s}^{\prime} \geq 0$, presentado en las figuras 2 y 3 , donde $D^{\prime}{ }_{\sigma}$ tiene el mismo valor. Esto confirma el efecto del campo $D^{\prime}$ s sobre la temperatura crítica del sistema.

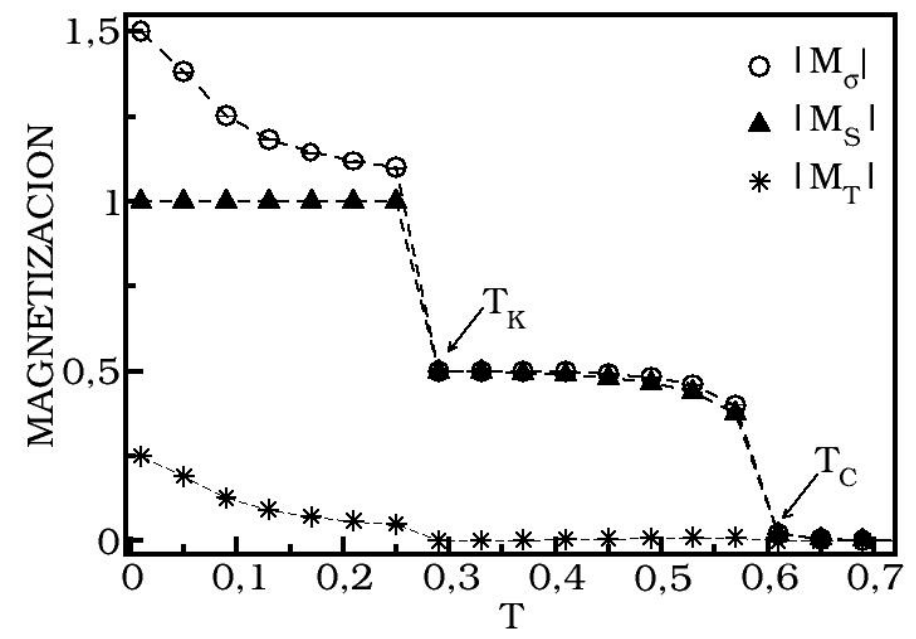

Fig. 6: Comportamiento de las magnetizaciones del sistema, $\left|M_{\sigma}(T)\right|,\left|M_{S}(T)\right|$ y $\left|M_{T}(T)\right| . T_{k}$ es la temperatura de la posible transición de primer orden. $D_{S}^{\prime}=-100 . D_{\sigma}^{\prime}=-1.95 . J_{1}<0$.

Estimamos las temperaturas críticas a través de la ubicación de los máximos de los calores específicos. En las figuras 7 y 8 , se presenta el comportamiento del calor específico del modelo para diversos valores negativos del campo $D_{\sigma}^{\prime}$, con $D_{s}^{\prime}=-3$ y $D_{s}^{\prime}=-1$, respectivamente.

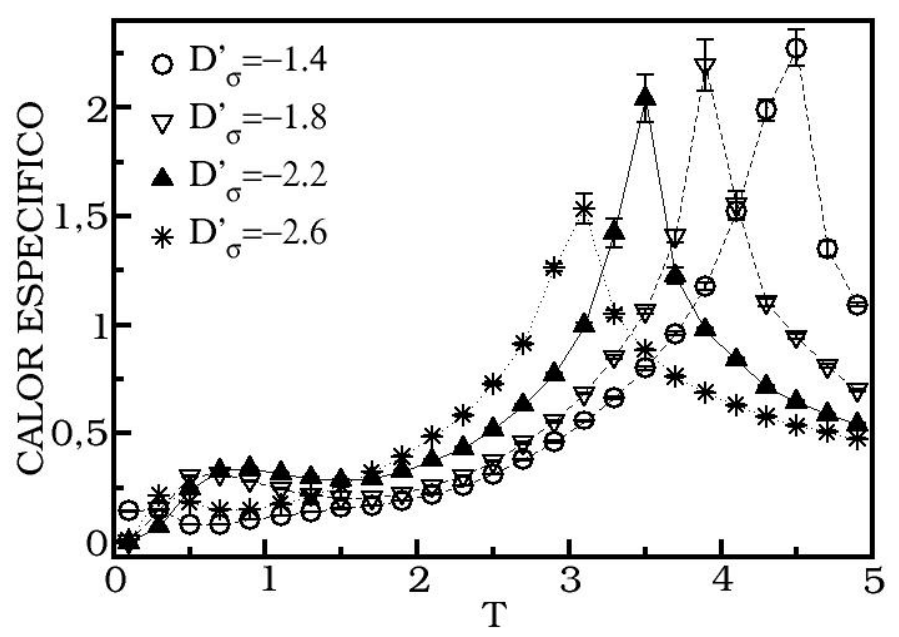

Fig. 7: Comportamiento del calor específico por espín del sistema, como una función de la temperatura. $D_{\sigma}^{\prime}<0 . D_{S}^{\prime}=3 . J_{1}<0$. 
Las gráficas nos muestran que, para un valor fijo de $D^{\prime} s$, y cuando $\left|D_{\sigma}\right|\left(D_{\sigma}^{\prime}<0\right)$ se incrementa, la $T_{C}$ disminuye. Las curvas de calor específico de la gráfica 7 , exhiben segundos picos a bajas temperaturas, los cuales se consideran independientes del tamaño de la red y no corresponden a puntos críticos (Selke y Oitmaa, 2010). La formación de estos máximos "anómalos", cuando $T \rightarrow 0$, surge cuando los espines de la subred $\sigma$ tratan de reorganizarse térmicamente debido al efecto de $D_{\sigma}$, ya que los espines tipo $S$ están más ordenados por la acción de $D_{S}$.

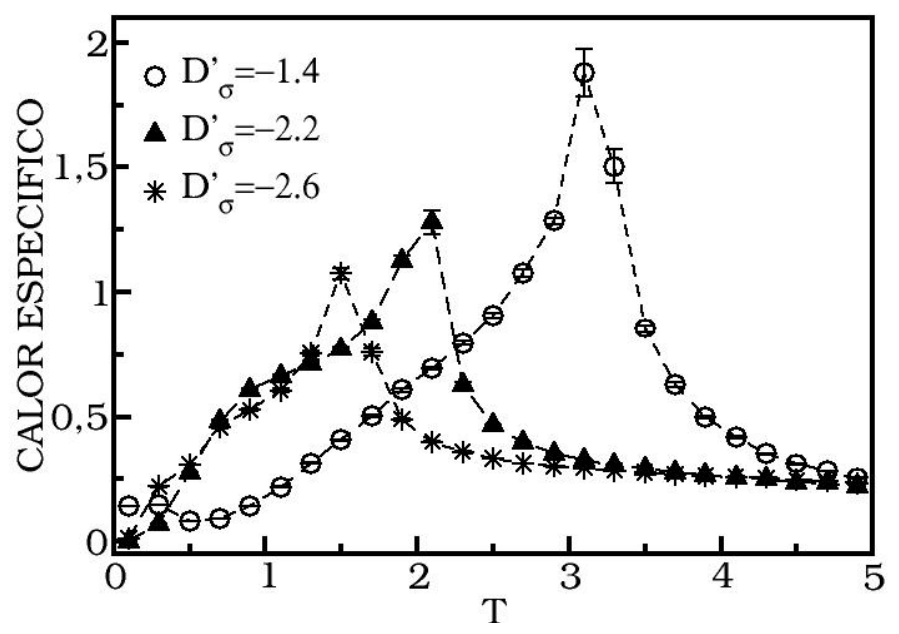

Fig. 8: Comportamiento del calor específico por espín del sistema, como una función de la temperatura. $D_{\sigma}^{\prime}<0 . D_{S}^{\prime}=-1 . J_{1}<0$.

Los resultados hallados, de la relación entre $D_{s}^{\prime}, D_{\sigma}^{\prime}, T_{\text {Comp }}$ y $T_{C}$, se resumen en las figuras 9 y 10 . En la gráfica 9 se exhibe el comportamiento de $T_{\text {Comp }}$ y $T_{C}$. Se observa, que $T_{C}$ es independiente de $D_{s}^{\prime}$ y $D_{\sigma}^{\prime}$, cuando $D_{s}^{\prime} \leq-2$, y alcanza un valor límite, que depende de $D_{\sigma}^{\prime}$, para valores grandes de $D^{\prime}$ s. Por encima de la curva de $T_{C}$, se encuentra la región paramagnética, y por debajo se tiene la región ferrimagnética. Las curvas de $T_{\text {Comp }}$ nos indican que, para los valores de $D_{\sigma}^{\prime}$, la región donde existen puntos de compensación es $D^{\prime} s \geq-1.6$. Además, para el rango de valores $1.99 \leq D_{\sigma}^{\prime} \leq-1.9$, una vez que aparece $T_{\text {Comp }}$, ésta es independiente de $D^{\prime}$ s, y alcanza rápidamente un valor límite. Para $D_{\sigma}^{\prime}=-2$ sólo hay $T_{\text {Comp }}$ en el rango $-1.6 \leq D^{\prime}{ }_{S} \leq 0$.

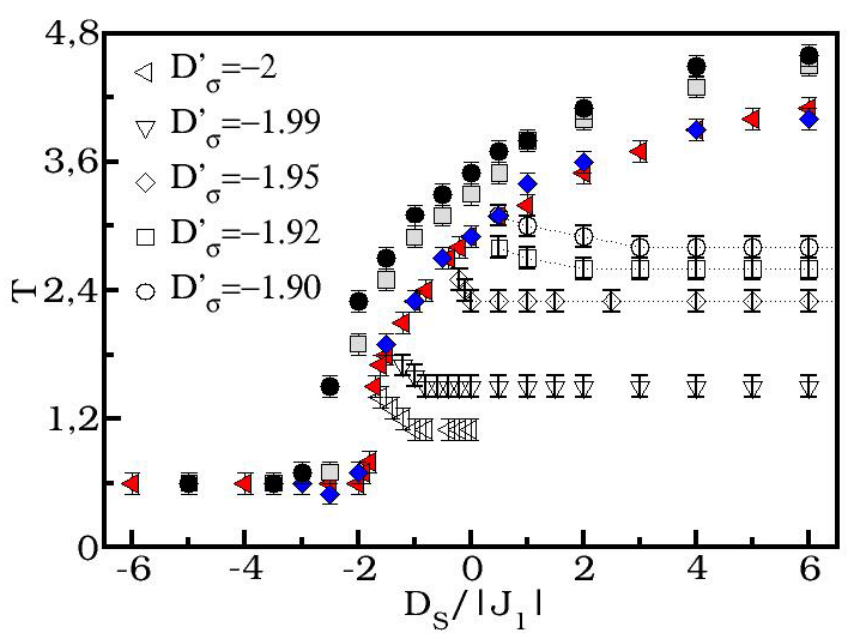

Fig. 9: Comportamiento de las temperaturas $T_{C}$ y $T_{\text {Comp. }}$. Para la $T_{C}$ (símbolos llenos): $D_{\sigma}{ }_{\sigma}=-1.5$ (círculos). $D_{\sigma}^{\prime}=-1.6$ (cuadrados). $D_{\sigma}^{\prime}=-1.95$ (rombos). $D_{\sigma}^{\prime}=-2.0$ (triángulos).

En la gráfica 10, sólo se muestra el comportamiento de la temperatura crítica. Para $D_{S}^{\prime}>0$, la $T_{C}$ aumenta con el incremento de $\left|D_{\sigma}^{\prime}\right|$; y al incrementarse $\left|D_{S}^{\prime}\right|$, para $D_{S}^{\prime}<0$, la $T_{C}$ decrece. En las figuras 9 y 10, las líneas punteadas se usan para visualizar las curvas, y las líneas continuas son ajustes lineales. 


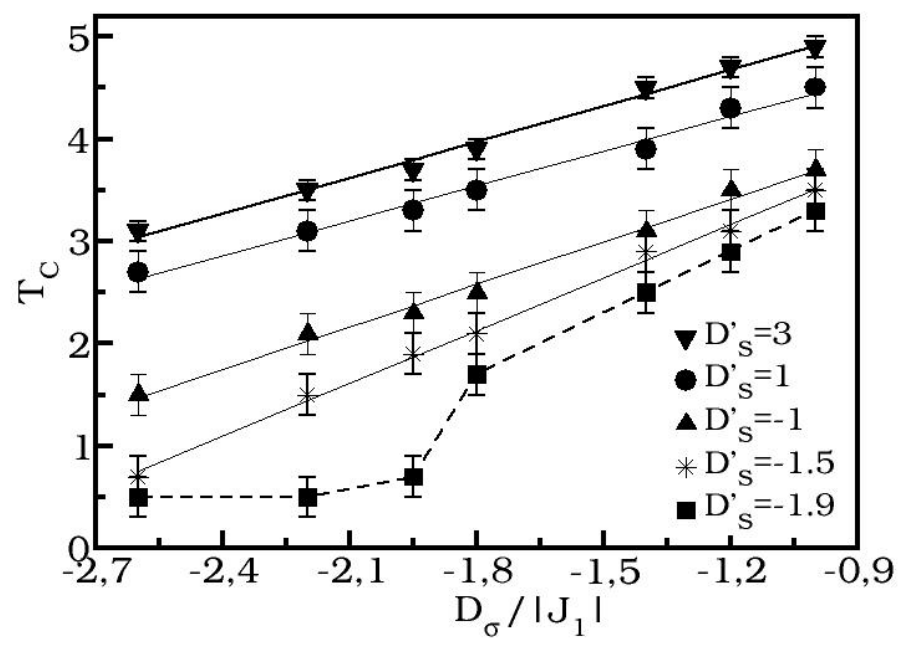

Fig. 10: Comportamiento de la temperatura crítica $\left(T_{C}\right)$ en función de los campos cristalinos $D_{s}^{\prime}$ y $D_{\sigma}^{\prime}$.

Los resultados obtenidos coinciden cualitativamente con los reportados por Albayrak e Yigit (Albayrak e Yigit, 2007), pero la técnica no perturbativa usada en nuestro trabajo, permite determinar con mayor precisión las regiones del espacio de parámetros en que aparecen las $T_{\text {Comp }}$. Como se esperaba, por el tipo de aproximación usada por Albayrak e Yigit (Albayrak e Yigit, 2007), las $T_{C}$ son menores en nuestro estudio, para los mismos valores de los campos $D^{\prime}$ y $D_{\sigma}^{\prime}$. Hecho que no es sorpresivo, puesto que es conocido que los métodos perturbativos sólo dan resultados aproximados para los modelos de Ising mixtos, e incluso, en trabajos realizados con teorías de campo medio sobre estos modelos, los mismos autores sugieren que sus resultados deben ser confirmados con métodos no perturbativos (Deviren et al., 2009a; Deviren y Keskin, 2010).

\section{CONCLUSIONES}

Se analiza, por medio de simulaciones de Monte Carlo, el comportamiento crítico de un sistema ferrimagnético de Ising de espines mixtos $S= \pm 3 / 2, \pm 1 / 2$ y $\sigma= \pm 5 / 2, \pm 3 / 2, \pm 1 / 2$, con diferentes anisotropías de ión simple, $D^{\prime} s$ y $D_{\sigma}^{\prime}$, que actúan sobre las subredes $S$ y $\sigma$, respectivamente. Se calculan los diagramas de fase a temperatura finita de la magnetización total, las magnetizaciones de las subredes y el calor específico, indicando las temperaturas de compensación y crítica para diversas combinaciones de los parámetros en el Hamiltoniano. Cuando el Hamiltoniano incluye interacciones antiferromagnéticas entre primeros vecinos y los campos de anisotropía están en los rangos $-2 \leq D_{\sigma}^{\prime} \leq-1.9$ y $D^{\prime}{ }_{S} \geq-1.6$, el sistema presenta temperaturas de compensación, fenómeno que se analizó en detalle mediante el comportamiento de las magnetizaciones de las subredes. El modelo también exhibe $T_{\text {Comp }}$ para $D_{S}^{\prime}=0$, lo que demuestra que $D^{\prime}{ }_{\sigma}$ es el principal responsable de la aparición del fenómeno de compensación. Para un valor fijo de $D_{\sigma}^{\prime}$, al incrementarse el campo $D_{s}^{\prime}$ se produce un incremento significativo de la temperatura crítica, lo que implica que el sistema sea más estable en su estado ferrimagnético. Nuestro estudio sugiere que existe una fuerte dependencia entre $T_{\text {Comp }}, T_{C}$ y los parámetros de campo cristalino. Para valores grandes del módulo $\left|D_{s}^{\prime}\right|\left(D_{s}^{\prime}<0\right)$, el modelo presenta posibles transiciones de fase de primer orden, dado que las magnetizaciones de las subredes decrecen discontinuamente, para un determinado valor de la temperatura.

\section{REFERENCIAS}

Albayrak, E. y A. Yigit, The phase diagrams of de mixed spin-3/2 and spin-5/2 Ising system on the Bethe lattice, Physica status solidi b: 244, 748-758 (2007).

Albayrak, E. y A. Yigit, Mixed spin-3/2 and spin-5/2 Ising system on the Bethe lattice, Physics Letters A: 353, 121-129 (2006). 
Behera, J. N. y otros 3 autores, Synthesis and characterization of ruthenium and iron-ruthenium prussian blue analogues, Chemistry of Materials: 21, 1922-1926 (2009).

Blundell, S. J. y F. L. Pratt, Organic and molecular magnets, Journal of Physics: Condensed Matter: 16, 771-828 (2004).

Buendía, G. y E. Machado, Magnetic Behavior of an Ising ferrimagnetic model in an oscillating field, Physical Review B: 61, 14686 (2000).

Buendía, G. y J. Villarroel, Compensation temperatures of mixed ferro-ferrimagnetic ternary alloys, Journal of Magnetism and Magnetic Materials: 310, E495-E497 (2007).

Coronado, E. y D. Gatteshi, Trends and challenges in molecule-based magnetic materials, Journal of materials chemistry: 16, 2513-2515 (2006).

De La Espriella, N. y G. Buendía, Ground state phase diagrams for the mixed Ising $3 / 2$ and $5 / 2$ spin model, Physica A: 389, 2775-2732 (2010).

De La Espriella, N. y G. Buendía, Magnetic behavior of a mixed Ising $3 / 2$ and $5 / 2$ spin model, Journal of Physics: Condensed Matter: 23, 176003-176009 (2011).

Deviren, B., M. Keskin y O. Canko, Magnetic properties of an anti-ferromagnetic and ferrimagnetic mixed spin-1/2 and spin-5/2 Ising model in the longitudinal magnetic field within the effective-field approximation, Physica A: 388, 1835-1848 (2009a).

Deviren B., M. Keskin y O. Canko, Kinetic Ising model in a time-dependent oscillating external magnetic field: Effective-field theory, Journal of Magnetism and Magnetic Materials: 321, 458-466 (2009b).

Deviren, B. y M. Keskin, Dynamic phase transitions and compensation temperatures in a mixed spin-3/2 and spin-5/2 Ising system, Journal of Statistical Physics: 140, 934-947 (2010).

Ekiz, C., Ferrimagnetism in the mixed spin Ising system on a two-fold Cayley tree, Journal of Magnetism and Magnetic Materials: 293, 759-767 (2005).

Ekiz, C., Influence of anisotropic crystal field on a ferrimagnetic mixed spin bilayer system, Physica A: 387, 1185-1199 (2008).

Figueiredo, W., M. Godoy y V. S. Leite, Compensation temperature of the mixed-spin Ising model on the hexagonal lattice, Brazilian Journal Physics: 34, 392-394 (2004).

Gatteshi, D. y R. Sessoli, Molecular nanomagnets: the first 10 years, Journal of magnetism and magnetic materials: 272-276, 1030-1036 (2004).

Htoutou, K., A. Ainane y M. Saber, The tranverse cristal-field effects of the mixed spin Ising bilayer system, Journal of Magnetism and Magnetic Materials: 269, 245-248 (2004).

Hurtado, N. y G. Buendía, Efecto del campo cristalino en un ferrimagneto mixto de Ising tridimensional, Revista Mexicana de Física: 49(3), 183-185 (2003).

Karl, G., Proof of a Conjecture by Luttinger and Tisza, Physical Review B: 7, 2050-2053 (1973).

Keskin, M. y M. Ertas, Existence of a dynamic compensation temperature of a mixed spin-2 and spin-5/2 Ising ferrimagnetic system in an oscillating field, Physical Review E: 80, 061140 (2009). 
Keskin, M. y E. Kantar, Dynamic compensation temperatures in the mixed spin-1 and spin-3/2 Ising system under a time-dependent oscillating magnetic field, Journal of Magnetism and Magnetic Materials: 322, 2789 (2010).

Keskin, M., e Y. Polat, Phase diagrams of a nonequilibrium mixed spin-3/2 and spin-2 Ising system in an oscillating magnetic field, Journal of Magnetism and Magnetic Materials: $321,3905-3912$ (2009).

Kiwi, M., Exchange bias theory, Journal of Magnetism and Magnetic Materials: 234, 584-595 (2001).

Li, P. F., Y. G. Cheng y H. Cheng, Second- to first- order transition in two coupled antiferromagnetic rings, European Physical Journal B: 51, 473 (2006).

Selke, W. y J. Oitmaa, Monte Carlo study of mixed-spin $S=(1 / 2,1)$ Ising ferrimagnets, Journal of Physics: Condensed Matter: 22, 076004 (2010).

Weiss, R., A. Gold y J. Terner, Cytochromes $c^{\prime}$ : Biological Models for the $S=3 / 2,5 / 2$ Spin-State Admixture?, Chem. Rev.: 106 (6), 2550-2579 (2006).

Yessoufou, R. A., S. H. Amoussa y F. Hontinfinde, Magnetic properties of the mixed spin-5/2 and spin-3/2 Blume-Capel Ising system on the two-fold Cayley tree, Central European Journal of Physics: 7(3), 555-567 (2009).

Zaim, A., M. Kerouad e Y. Belmamoun, Monte Carlo study of a mixed spin-1/2 and spin-1 BlumeCapel ferrimagnetic model with four-spin interaction, Physica B: 404, 2280-2284 (2009).

Zhang, Q., G. Wei e Y. Gu, The study of the phase diagram and internal energy of the mixed spin$3 / 2$ and spin-5/2 ferrimagnetic Ising system with interlayer coupling by effective-field theory; a simple approach of calculating internal energy, Physica status solidi b: 242 (4), 924-932 (2005).

Zukovic, M. y A. Bobak, Phase diagram of a mixed spin-1 and spin-3/2 Ising ferrimagnet, Physica A: 389, 5402-5407 (2010). 
